\title{
TWO NEW PROOFS OF THE TEST PARTICLE SUPERPOSITION PRINCIPLE OF PLASMA KINETIC THEORY
}

BY

J. A. KROMMES

\section{PLASMA PHYSICS LABORATORY}

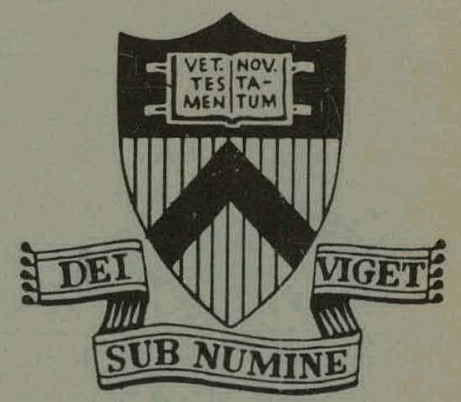

DISTRUBUTION OF THIS DOCUMENT IS UNUIMUTED

\section{PRINCETON UNIVERSITY PRINCETON, NEW JERSEY}

This work was supported by U. S. Energy Research and Development Administration Contract $\mathrm{E}(11-1)-3073$. Reproduction, translation, publication, use and disposal, in whole or in part, by or for the United States Government is permitted. 


\section{DISCLAIMER}

This report was prepared as an account of work sponsored by an agency of the United States Government. Neither the United States Government nor any agency Thereof, nor any of their employees, makes any warranty, express or implied, or assumes any legal liability or responsibility for the accuracy, completeness, or usefulness of any information, apparatus, product, or process disclosed, or represents that its use would not infringe privately owned rights. Reference herein to any specific commercial product, process, or service by trade name, trademark, manufacturer, or otherwise does not necessarily constitute or imply its endorsement, recommendation, or favoring by the United States Government or any agency thereof. The views and opinions of authors expressed herein do not necessarily state or reflect those of the United States Government or any agency thereof. 


\section{DISCLAIMER}

Portions of this document may be illegible in electronic image products. Images are produced from the best available original document. 
This report was prepared as an account of work sponsored by the United States Government. Neither the United States nor the United States Energy Research and Development Administration, nor any of their employees, nor any of their contractors, subcontractors, or their employees, makes any warranty, express or implied, or assumes any legal liability or responsibility for the accuracy, completeness or usefulness of any information, apparatus, product or process disclosed, or represents that its use would not infringe privately owned rights.

Printed in the United States of America.

Available from

National Technical Information Service

U. S. Department of Commerce 5285 Port Royal Road

Springfield, Virginia 22151

Price: Printed Copy \$ * ; Microfiche $\$ 1.45$

*Pages

$1-50$

$51-150$

$151-325$

$326-500$

501-1000
NTIS

Selling Price

$\$ 4.00$

5.45

7.60

10.60

13.60 
Two New Proofs of the Test Particle Superposition Principle of Plasma Kinetic Theory

John A. Krommes*

Plasma Physics Laboratory, Princeton University Princeton, New Jersey 08540

ABSTRACT

The test particle superposition principle of plasma kinetic theory is discussed in relation to the recent theory of two-time fluctuations in plasma given by Williams and Oberman. Both a new deductive and a new inductive proof of the principle are presented; the deductive approach appears here for the first time in the literature. The fundamental observation is that two-time expectations of one-body opera- . tors are determined completely in terms of the $(\underset{\sim}{x}, \underset{\sim}{v})$ phase space density autocorrelation, which to lowest order in the discreteness parameter obeys the linearized vlasov equation with singular initial condition. For the deductive proof, this equation is solved formally using time-ordered operators, and the solution then rearranged into the superposition principle. The inductive proof is simpler than Rostoker's, although similar in some ways; it differs in that first order equations for pair. correlation functions need not be invoked. It is pointed out that the superposition principle is also applicable to the short-time theory of neutral fluids. 


\section{! \\ I. INTRODUCTION}

The principle of superposition of dressed test particles ${ }^{l}$ has been of great conceptual importance and practical utility in the kinetic theory of plasma physics, as it provides an algorithm for the computation of certain one- or two-time expectations which is both mathematically simple and physically revealing. The principle was first established rigorously by Rostoker, I allivugh examples of the principlc had been given previously. $\%, 3$ In words, the theorem states that to first order in the discreteness parameter (plasma parameter) $\varepsilon_{\mathrm{p}} \equiv 1 / \mathrm{n} \lambda_{\mathrm{D}} \mathrm{d}$, (with $\mathrm{n}$ the density, $\lambda_{D}$ the Debye lenqth, and $d$ the numher of dimoncions), the plaslld may be treated as a collection of uncorrelated quasiparticles-noninteracting point particles combined ("dressed") with the lowest order Vlasov response to them. The purpose of this report is to show that the superposition principle is related simply to the kinetic theory of two-time fluctuations proposed recently by williams and oberman. 4 In doing so, we shall provide two new, compact, and lucid proofs of the principle. Our first proof is deductive in nature, thus differing in philosophy from Rostoker's original inductive proof. A deductive proof is of considerable interest, as it emphasizes that the superposition principle follows naturally and readily from the general principles of plasma kinetic theory. In" contrast to this, the original proof of the principle was motivated only by particular examples which were sometimes mathematically complex and opaque. For completeness, we shall also give a new inductive proof which simplifies considerably Rostoker's original one. We shall deal immediately with the 
most general two-time version of the principle; the one-time result follows as a special case.

In discussing two-time fluctuations, a consistent convention for time arguments is essential. The one we have adopted aiffers from Rostoker's, and considerable confusion may result unless the following is understood clearly. There are essentially three times in the problem: the preparation time of the system, denoted by $t_{p}$; the initial measurement time $t_{0}$; and the final measurement time $t$. This is to be compared to Rostoker's notation, which is obtained from ours by the mapping

$$
t \rightarrow t^{\prime}, \quad t_{0} \rightarrow t, \quad t_{0}=t_{p} \rightarrow t=0
$$

Our notation is particularly convenient and emphatic for a discussion of two-time fluctuations. In two-time theory, almost all dynamical operators act at time $t$; our notation avoids a cluttered proliferation of primes or subscripts. In actual computations one deals mostly with one-sided functions (denoted by subscript "+") with $t \geq t_{0}$; this time sequence is easier to remember than $t^{\prime} \geq t$. Initial conditions on two-time functions will be imposed at $t_{0}$. It is vital to note that $t_{0}$ does not generally coincide with $t_{p}$; in fact, usually $t_{o}>t_{p}$ so that initial transients have died away. In particular, in thermal equilibrium situations we have $t_{p}=-\infty$, so that the initial conditions on the two-time quantities are the asymptotic long-time solutions of the appropriate one-time equations. For notational clarity, we do not write explicitly the dependence of all quantities on $t_{p} \cdot A$ 
further difference with Rostoker's notation is that the order of writing time arguments is reversed here: in our work later times appear to the left of earlier times, while in Rostoker's the reverse is true $\left(e \cdot g \cdot \Gamma_{+}\left(x, t ; x_{0}, t_{0}\right)\right.$ rather than $\left.\Gamma_{+}\left(x, t ; x^{\prime}, t^{\prime}\right)\right)$.

Define the phase point $X \equiv\left(\underset{w}{x}, \underset{\sim}{v}, s_{i}\right)$, with $\underset{\sim}{x}$ the position, $\underset{\sim}{v}$ the velocity, and $s$ the species label of a particle. Most work in plasma kinetic theory, including Rostoker's proof of the superposition principle!, has dealt with certain clemental two-linte probability functions: the test particle function $\Omega\left(x, t_{i} \dot{x}_{0}, t_{0}\right)$, the probability that a (test) particle of speries $s_{0}$ is at $\left({\underset{\sim}{O}}_{0}, v_{0}, t_{0}\right)$ and that that same particle is at $(\underset{\sim}{x}, \underset{\sim}{v}, t)$ (clearly $\Omega$ vanishes unless $\left.s=s_{0}\right)$; the field particle function $F\left(x, t_{i} x_{0}, t_{0}\right)$, the probability that a (test) particle of species $s_{0}$ is at $\left({\underset{\sim}{x}}_{0}, \vee_{0}, t_{0}\right)$ and that some different (field) particle of species $s$ is at $(x, y, t)$; and certain higher order functions. Generaily speaking, it is cumbersome to work directly with these functions, as they enter the fluctuation theories in only certain welldefined combinations, 5 their parts of dominant order in $\varepsilon_{p}$ cancel, and one must include complicated higher order terms in the theory to obtain nontrivial results. For example, if we define a onebody operator $A$ by the additive phase dependence

$$
A(X, t) \equiv \sum_{s} \sum_{i=1}^{N_{s}} a_{s}\left(x_{i} x_{i}(t)\right),
$$

with $\mathrm{N}_{\mathrm{s}}$ the total number of particles of species $\mathrm{s}$ and $\mathrm{x}_{i}(t)$ the exact phase trajectory of particle $i$, and also define the fluctuation $\delta A \equiv A-\langle A\rangle$, then it is easy to show 2,4 that the two-time 
expectation of the fluctuations of two such operators A,B becomes ${ }^{6}$

$$
\begin{aligned}
\left.<\delta A(X, t) \delta B\left(X_{0}, t_{0}\right)\right\rangle= & \sum_{s} n_{s} \sum_{s_{0}} n_{s_{0}} \int d Y d Y a(X ; Y) b\left(X_{0} ; Y_{0}\right) \\
& X\left[n_{s} \Omega\left(Y, t_{0} ; Y_{0}, t_{0}\right)+F\left(Y, t_{;} Y_{0}, t_{0}\right)\right. \\
& \left.-E(Y, t) f\left(Y_{0}, t_{0}\right)\right],
\end{aligned}
$$

where the important combination

$$
\Gamma \equiv \mathrm{n} \Omega+\mathrm{F}-\mathrm{ff}
$$

has appeared. If one writes separate evolution equations for $\Omega$, $F$, and $f$, and only later combines their solutions according to (3), one must treat the dynamics of $F$ to first order in $\varepsilon_{p}$ since F has a dominantly uncorrelated zeroth order part which cancels in the combination (3):

$$
\begin{aligned}
F\left(Y, t_{;} Y_{0}, t_{0}\right) & =f(Y, t) f\left(Y_{0}, t_{0}\right)+\bar{F}\left(Y, t_{;} Y_{0}, t_{0}\right) ; \\
\bar{F} & =o\left(\varepsilon_{p}\right) .
\end{aligned}
$$

This was Rostoker's original procedure. ${ }^{2}$ However, the combination $\Gamma$ is already $O\left(\varepsilon_{p}\right)$. A lowest order nontrivial result for (2) can therefore be obtained by treating the dynamics of $\Gamma$ to zeroth order in $\varepsilon_{\mathrm{p}}$; the size of $\Gamma$ enters only through the initial conditions $\Gamma\left(t_{0} ; t_{o}\right)=\left(\varepsilon_{p}\right)$. This is a substantial computational savings. Furthermore, to work with the elemental functions themselves rather than with the relevant comblnations of them is to obscure the appropriate generalizations of the kinetic theory to 
higher order, or more importantly the appropriate renormalization of the lowest order theory. This latter remark is of cons/iderable importance since it has been found that renormalized theories are vital in the correct treatment of certain situations such as the anomalous transport due to fluctuations long-lived on the vlasov scale. 7,8 : theories, it is very important to couch the lowest order theory as symmetrically and compactly as póssible.

Recently, a number of authors ${ }^{4,7-9}$ have proposed kinetic theories which overcome the objections raised in the preceding paragraph by dealing directly with the fundamental quantity $\Gamma$ and its generalizations. ( $\Gamma$ can be defined alternatively to (3) as

$$
\Gamma\left(x, t_{;} x_{0}, t_{0}\right)=\left\langle\hat{\delta N}(x, t) \delta \hat{N}\left(x_{0}, t_{0}\right)>\right.
$$

where $\delta \hat{N}$ is the fluctuation in the Klimontovich microdensity $\hat{N}$ :

$$
\hat{N}(x, t)=\frac{1}{n} \sum_{i=1}^{N} \delta\left[x-x_{i}(t)\right],
$$

with $\langle\hat{N}\rangle=f(X, t)$ the one body distribution.) In particulär, Williams and oberman ${ }^{4}$ showed that $\Gamma \equiv \Gamma^{(1)}$ is the first member of a set of two-time quantities $\left\{\bar{\Gamma}^{(r)}\left(x_{1}, x_{2}, \ldots, x_{r} ; t ; x_{0}, t_{0}\right) \mid r=1,2, \ldots\right\}$ which collectively obeys the linearized BBGKY cumulant hierarchy (in the $t$ variables) with certain partially singular initial conditions successively smaller in $\varepsilon_{p}: \bar{\Gamma}^{(r)}\left(t_{0} ; t_{0}\right)=O\left(\varepsilon_{p}^{r}\right) \cdot{ }^{: A} A$ detailed account of this kinetic theory has been given elsewhere. 7,8 
However, to discuss the relation of this theory to the test particle superposition principle, we need here only the lowest order result (denoted by subscript "O"), which is already well-known to workers in the field of classical fluids ${ }^{10}$ : $\Gamma_{0}$ satisfies the linearized Vlasov equation with singular initial condition

$$
\begin{aligned}
\Gamma_{0}\left(x, t_{0} ; x_{0}, t_{0}\right) & \equiv \xi\left(x, x_{0}, t_{0}\right) \\
& =n^{-1} \delta\left(x-x_{0}\right) f\left(x_{0}, t_{0}\right)+g_{0}\left(x, x_{0}, t_{0}\right) .
\end{aligned}
$$

Here $f$ is the one body function which solves the nonlinear timedependent Vlasov equation, and $g_{0}$ is the irreducible pair correlation function, or two body cumulant, computed to first order in $\varepsilon_{\mathrm{p}}$ (that is, to lowest nontrivial order). The lowest order, collisionless ${ }^{11}$ result for two-time expectations can then be transcribed directly from (2) as

$$
\begin{aligned}
& \left\langle\delta A(X, t) \delta B\left(X_{0}, t_{0}\right)\right\rangle_{0} \\
& \quad-\sum m_{0} \int d Y d y_{0} d(X ; Y) i_{0}\left(Y, t ; Y_{0}, t_{0}\right) b\left(X_{0} ; Y_{0}\right) .
\end{aligned}
$$

By contrast, the superposition principle ${ }^{l}$ computes $\langle\delta A \delta B\rangle$ as

$$
\begin{aligned}
& <\delta A(x, t) \delta B\left(x_{0}, t_{0}\right)>>_{0} \\
& =\sum n^{\prime} n^{\prime \prime} \int d X^{\prime} d X^{\prime \prime} a\left(x ; x^{\prime}, t\right) w\left(x^{\prime}, t ; x^{\prime \prime}, t_{0}\right) B\left(x_{0} ; x^{\prime \prime}, t_{0}\right),
\end{aligned}
$$

whcre $\hat{a}$ and $\hat{B}$ are certain quasiparticle densities to be defined 
mathematically below, and $W \equiv n \Omega_{0}$ is the collisionless streaming solution for the evolution of a test particle.

Since both approaches (7) and (8) to two-time fluctuations consider only lowest order effects in $\varepsilon_{p}$, it is in one sense "intuitively obvious" that their predictions must be identical. However, the mathematical formulations and their physical interpretations are superficially quite distinct. Physically speaking, for example, (7) with (6) describes $\left\langle\delta A(t) \delta R\left({ }_{0}\right)\right\rangle$ as a two-stop measurement process performed on a correlated plasma. The first measurement is performed at $t_{0}$, when the plasma is "caught" in the correlated state $\xi\left(t_{0}\right)$. This measurement effectively disturbs the plasma and the disturbance propagates as a Vlasov fluctuation to time $t$, when the second measurement is performed. On the other hand, the two-step measurement described by (8) is performed on a system of uncorrelated quasiparticles. The disturbance in the quasiparticles induced by the first measurement streams (no selfconsistent response) to time $t$. That these two processes are equivalent is not obvious; furthermore, the mathematics of neither approach resembles that of the original procedure ${ }^{2}$ beginning from (2) and separate evolution equations for $\Omega, F$, and $f$. An explicit demonstration of the equivalence between the formalisms is therefore desirable. Furthermore, the original proof of the superposition principle is somewhat unsatisfying, as the result is postulated inductively, then verified. Although the induction hypothesis is well-founded, as the principle has been verified in certain detailed (and mathematically complicated) examples, it is nevertheless desirable to give a deductive proof of the principle. 
Such an approach demonstrates much more convincingly the intimate relation between the superposition principle and the general (lowest order) structure of plasma kinetic theory.

In the remainder of the work we shall give one deductive and one inductive proof of the superposition principle, beginning from (7). We will see that our proofs are more compact than was Rostoker's original one. In fact, Rostoker had to invoke the second member of the BBGKY hierarchy for $g_{0}$, as well as its moderately complicated two-time generalization. However, our more judicious choice of $\Gamma$ as fundamental quantity allows us to deal with nothing more complicated than the Vlasov equation. This simplification lends strong support to our assertion that $\Gamma$, rather than $\Omega$ or $F$, is the appropriate kinetic theoretic object with which to deal, and agrees with our intuitive notion of the plasma as dominantly vlasov in character.

The organization of the paper is as follows. In section II we introduce an appropriate notation, state the superposition principle in terms of it, and formulate the mathematical assertion which must be proved. In section III we give for the first time a deductive proof of the principle by using simple facts about time-ordered operators to rearrange appropriately the formal solution of the Vlasov equation. In section IV we prove the principle Inductively by posiülāting the result, then showing its equivalence to the $\Gamma$ formalism (7). In section $V$ we comment briefly on the applicability of the superposition principle to the theory of neutral fluids, and discuss and summarize our results. 


\section{NOTATION. FORMULATION OF THE PROBLEM}

We begin by introducing a suitable notation. Henceforth, we shall omit the "o" superscript as we deal only with lowest order phenomena. As did Rostoker, we restrict ourselves to electrostatic phenomena only; we will discuss electromagnetic extensions of the theory. in a future paper. The Landau operator $L$ is now defined by writting the linearized Vlasov equation as

$$
\left(\partial_{t}+i L\right) \Gamma=0
$$

Thus, $L$ consists of a streaming term $S$ and a Vlasov term $V$ :

$$
\begin{aligned}
& \left.i S \equiv \underset{\sim}{\underset{v}{v}} \underset{\sim}{\nabla}+\underset{\sim}{F} \operatorname{ext}(X, t) \cdot \frac{\partial}{\partial \underset{\sim}{v}}+\underset{\sim}{E f}\right) \cdot \underset{\sim}{\partial}, \\
& i V \equiv(\partial f) \cdot \underline{\sim},
\end{aligned}
$$

and

$$
\begin{aligned}
& \underset{\sim}{\partial} \equiv\left(\frac{q}{m}\right) \frac{\partial}{\partial \underset{v}{v}}
\end{aligned}
$$

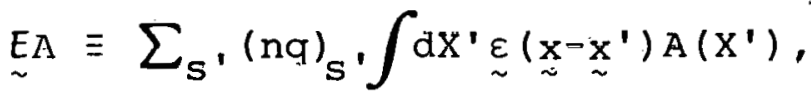

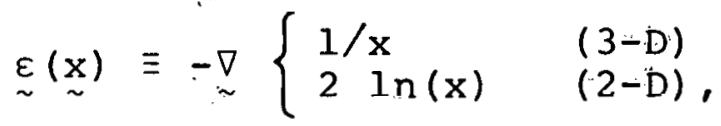

$$
\begin{aligned}
& \underset{\sim}{\mathrm{F}} \operatorname{ext} \equiv \mathrm{q}_{\sim}^{\mathrm{E}} \operatorname{ext} \underset{\sim}{(x, t)}+\Omega_{C} \underset{\sim}{(x, t)} \underset{\sim}{\mathrm{v}} \mathrm{x} \underset{\sim}{(\mathrm{B} / \mathrm{B})}, \\
& \Omega_{c} \equiv q B(x, t) / m c .
\end{aligned}
$$

Note that $L$ can be in general a function of time, both explicitly 
through $\underset{\sim}{F}$ ext $(X, t)$ and implicitly through the functional dependence on $f$.

In terms of the above notation, the quasiparticle density $\hat{a}$ is now defined as ${ }^{1}$

$$
\hat{a}\left(X ; X^{\prime}, t\right) \equiv \sum n \int d Y a(X ; Y) \gamma\left(Y, t \mid X^{\prime}\right),
$$

where

$$
Y\left(Y, t \mid X^{\prime}\right) \equiv n^{-1} \delta\left(Y-X^{\prime}\right)+P\left(Y, t \mid X^{\prime}\right)
$$

and $\mathrm{P}$ obeys the test particle equation:

$$
\begin{aligned}
\left(\partial_{t}+i L+i S^{\prime}\right) P\left(Y, t \mid X^{\prime}\right) & =-q \underset{\sim}{\varepsilon}\left(\underset{\sim}{y}-\underset{\sim}{x^{\prime}}\right) \cdot\left[\underset{\sim}{\partial} f^{\prime} f\left(X^{\prime}, t\right)\right] \\
& =-i V n^{-1} \delta\left(Y-X^{\prime}\right) .
\end{aligned}
$$

The S' term describes the streaming of the test particle represented by the $\delta\left(Y-X^{\prime}\right)$ term; the $L$ term describes the Vlasov response to that test particle. Since $Y\left(Y, t \mid X^{\prime}\right)$ is the fundamental quantity which appears in (10), it is convenient to replace (12) with an equation for $\gamma$. This is readily accomplished. From (12),

$$
\begin{aligned}
\left(\partial_{t}+\right. & \left.i L+i S^{\prime}\right) \gamma\left(Y, t \mid X^{\prime}\right) \\
& =\left(i L+i S^{\prime}\right) n^{-1} \delta^{\prime}\left(Y-X^{\prime}\right)-i V_{i 1}^{-1} \delta\left(Y-Y^{\prime}\right) \\
& =\left(i S+i S^{\prime}\right) n^{-1} \delta\left(Y-X^{\prime}\right) .
\end{aligned}
$$

Since $S$ is a differential operator in the $\underset{\sim}{(x, v)}$ phase space,

$$
S \delta\left(Y-X^{\prime}\right)=-S^{\prime} \delta\left(Y-X^{\prime}\right)
$$


and (13) becomes

$$
\left(\partial_{t}+i L+i S^{\prime}\right) \gamma\left(Y, t \mid X^{\prime}\right)=0
$$

The appropriate initial condition is

$$
\gamma\left(Y, t_{p} \mid X^{\prime}\right)=n^{-1} \delta\left(Y-X^{\prime}\right)+P\left(Y, t_{p} \mid X^{\prime}\right)
$$

for the moment, we do not specify $P\left(t_{p}\right)$. The motion of the test particle ( $S^{\prime}$ ) and its shielding cloud ( $L$ ) is particularly evident in the form (15a).

In terms of $\gamma$, the superposition principle (8) reads $?$

$$
\begin{aligned}
& <\delta A(X, t) \delta B\left(X_{O}, t_{O}\right)> \\
& =\sum n n_{C} \int d Y d Y_{O} a(X ; Y) b\left(X_{O} ; Y_{O}\right) \\
& \quad x \sum n^{\prime} n^{\prime \prime} \int d X^{\prime} d X^{\prime \prime} Y\left(Y, t \mid X^{\prime}\right) W^{\prime}\left(X^{\prime}, t ; X^{\prime \prime}, t_{O}\right) Y\left(Y_{O}, t_{O} \mid X^{\prime \prime}\right),
\end{aligned}
$$

where $W\left(X^{\prime} t_{;} X^{\prime \prime} t_{0}\right)$. obeys the streaming equation

$$
\begin{aligned}
& \left(\partial_{t}+i S^{\prime}\right) w^{\prime}\left(X^{\prime}, t_{i} x^{\prime \prime}, t_{0}\right)=0 \text {; } \\
& W\left(X^{\prime}, t_{0} ; X^{\prime \prime}, t_{0}\right)=\left(n^{\prime}\right)^{-1} \delta\left(X^{\prime}-x^{\prime \prime}\right) f\left(x^{\prime \prime}, t_{0}\right) .
\end{aligned}
$$

Comparing (16) with (7) and noting that $A$ and $B$ are arbitrary, we see that to prove the superposition principle is to prove that to lowest order in $\varepsilon_{\mathrm{p}}$ 


$$
\begin{aligned}
& \Gamma\left(Y, t_{;} Y_{0}, t_{0}\right)=\tilde{\Gamma}\left(Y, t_{;} Y_{0}, t_{0}\right) \\
& \equiv \sum n^{\prime} n^{\prime \prime} \int d X^{\prime} d X^{\prime \prime} Y\left(Y, t \mid X^{\prime}\right) W\left(X^{\prime}, t_{;} X^{\prime \prime}, t_{0}\right) Y\left(Y_{0}, t_{O} \mid X^{\prime \prime}\right) .
\end{aligned}
$$

We shall of course need the initial condition (6) for $\Gamma$. This initial condition contains the pair correlation function $g\left(X, x_{0}, t_{0}\right)$, which obeys the second member of the one-time BBGKY hierarchy--truncated, in this lowest order case, by the neglect of the three-body cumulant. However, $g$ is needed only in the particular combination $\xi$; furthermore, it is needed only to first order in $\varepsilon_{\mathrm{p}}$. In these circumstances, it is not necessary to invoke the cumulant equation for $g$ as did Rostoker; $\xi$ can be determined directly from the dynamical equation (9) for $\Gamma$. Thus,

$$
\begin{aligned}
\frac{\partial}{\partial t_{0}} \xi\left(Y, Y_{0}, t_{0}\right)= & \left.\frac{\partial}{\partial t} \Gamma\left(Y, t_{;} Y_{0}, t_{0}\right)\right|_{t=t_{0}} \\
& +\left.\frac{\partial}{\partial t_{0}} \Gamma\left(Y, t_{;} Y_{0}, t_{0}\right)\right|_{t=t_{0}} .
\end{aligned}
$$

We use the symmetry

$$
\Gamma\left(Y, t_{i} Y_{0}, t_{0}\right)=\Gamma\left(Y_{O}, t_{0} ; Y, t\right),
$$

apparent from (4), to write (19) as

$$
\begin{aligned}
\frac{\partial}{\partial t_{0}} \xi\left(Y, Y_{0}, t_{0}\right)= & -\left.i L \Gamma\left(Y, t_{;} Y_{0}, t_{0}\right)\right|_{t}=t_{0} \\
& -\left.i L_{0} \Gamma\left(Y_{0} ; t_{0} ; Y, t\right)\right|_{t}=t_{0}
\end{aligned}
$$


or

$$
\left(\partial_{t_{0}}+i L+i L_{O}\right) \xi\left(Y, Y_{O}, t_{O}\right)=0
$$

with initial condition

$$
\xi\left(Y, Y_{O}, t_{p}\right)=n^{-1} \delta\left(Y-Y_{O}\right) \dot{f}\left(Y_{0}, t_{p}\right)+g\left(Y, Y_{0}, t_{p}\right) \text {. }
$$

We will discuss the initial condition on $g$ shortly.

This concludes the preparatory formulation of the problem. We now turn to the proofs.

\section{THE DEDUCTIVE PROOF}

In this section, we give a deductive proof of the superposition principle (18) which begins from the formal solution of (9). In the general case, (9) is complicated because of the arbitrary space-time dependence of $\underset{\sim}{\mathrm{F}}$ ext and $f$, and this complication manifests itself immediately when the solution of a particular problem is attempted. Nevertheless; it is possible to make progress with the formal solution; in fact, the problem can be reduced essentially to the special case of time-independent $\underset{\sim}{\text { Fext }}$ and $f$ by the appropriate use of time-ordered exponentials. The time-ordering " + " of two operators $\hat{a}$ and $\hat{b}$ is defined by

$$
\begin{aligned}
\left\{\hat{a}\left(t_{1}\right) \hat{b}\left(t_{2}\right)\right\}+:= & H\left(t_{1}-t_{2}\right) \hat{a}\left(t_{1}\right) \hat{b}\left(t_{2}\right) \\
& +H\left(t_{2}-t_{1}\right) b\left(t_{2}\right) \hat{a}\left(t_{1}\right),
\end{aligned}
$$


where $\mathrm{H}$ is the Heaviside function; the time-ordered exponential is defined in terms of this by

$$
\begin{aligned}
\exp & \left\{\int_{0}^{t} d t^{\prime} \hat{a}\left(t^{\prime}\right)\right\} \equiv \sum_{n=0}^{\infty} \frac{1}{n !}\left[\int_{0}^{t} d t^{\prime} \hat{a}\left(t^{\prime}\right)\right]_{+}^{n} \\
& =\sum_{n=0}^{m}\left\{\int_{0}^{t} d t_{1} \int_{0}^{t_{1}} d t_{2} \ldots \int_{0}^{\left.t_{n-1} d t_{n} \hat{a}\left(t_{1}\right) \hat{a}\left(t_{2}\right) \ldots \hat{a}\left(t_{n}\right)\right\} .}\right.
\end{aligned}
$$

With these definitions, the solution of

$$
\left[\partial_{t}-\hat{a}(t)\right] \psi(t)=0 \quad\left(t>t_{0}\right)
$$

becomes

$$
\psi(t)=\exp _{+}\left\{\iint_{t_{0}}^{t} d t^{\prime} \hat{a}\left(t^{\prime}\right)\right\} \psi\left(t_{0}\right) \quad\left(t>t_{0}\right),
$$

which reduces to the familiar result

$$
\psi(t)=\exp \left[\left(t-t_{0}\right) \hat{a}\right] \psi\left(t_{0}\right) \quad\left(t>t_{0}\right)
$$

for time-independent $\hat{a}$. We shall shortly make use of the formal similarity between (24a) and (24b). Another result of use to us is the property 


$$
\left.\exp _{+}\left\{\int_{0}^{t} d t^{\prime}\left[\hat{a}\left(t^{\prime}\right)+\hat{b}\left(t^{\prime}\right)\right]\right\}=\exp +\left\{\int_{0}^{t} d t^{\prime} \hat{a}\left(t^{\prime}\right)\right\} x \exp +\int_{0}^{t} d t^{\prime} b\left(t^{\prime}\right)\right\}
$$

which is easily verified from (22).12

We wish the following proof to be as pedagogical and uncluttered as possible. Therefore, we shall write all formulas for the special case of time-independent background, using the simple exponential notation (24b). This case is of considerable interest in itself; it covers, for example, the important case of two-time fluctuations in thermal equilibrium, and more generally any situation in which $f$ varies slowly relative to the plasma time scale on which f evolves. Nevertheless, the results (23)-(25) assure us that the proof of the general case can be recovered immediately by replacing all simple exponentials by time-ordered ones according to

$$
\exp (\hat{a} t) \rightarrow \exp +\left\{\int_{0}^{t} d t^{\prime} \hat{a}\left(t^{\prime}\right)\right\}
$$

We shall actually provide the proof of (18) by dealing with the one-sided function

$$
\Gamma_{+}\left(x, t ; x_{0}, t_{0}\right) \equiv H\left(t-t_{0}\right) \Gamma\left(x, t ; x_{0}, t_{0}\right)
$$

and similarly $\mathrm{W}_{+}{ }^{\cdot}$. This is sufficient, as the two-sided functions are determined uniquely from the one-sided ones; e.g.,

$$
\Gamma\left(x, t_{;} x_{0}, t_{0}\right)=\Gamma_{+}\left(x, t_{;} x_{0}, t_{0}\right)+\Gamma_{+}\left(x_{0}, t_{0} ; x, t\right) .
$$


Consider, then, the Green's function solution of (9) for $\Gamma_{+}$:

$$
\Gamma_{+}\left(Y, t_{i} Y_{0}, t_{0}\right)=\sum_{S^{\prime}} \int d X^{\prime} U_{+}\left(Y, t^{\prime} X^{\prime}, t_{0}\right) \xi\left(X^{\prime}, Y_{0}, t_{0}\right)
$$

where we have introduced the retarded vlasov propagator

$$
u_{+}\left(Y, t ; X^{\prime}, t_{0}\right) \equiv H\left(t-t_{0}\right) \exp \left[-i L\left(t-t_{0}\right)\right] \delta\left(Y-X^{\prime}\right) .
$$

Similarly, we find from (20)

$$
\begin{aligned}
& \xi\left(X^{\prime}, Y_{0}, t_{0}\right) \\
& \quad=\exp \left[-i\left(L^{\prime}+L_{0}\right) t_{0}\right]\left(n^{\prime}\right)^{-1} \delta\left(X^{\prime}-Y_{0}\right) f\left(Y_{0}\right),
\end{aligned}
$$

where for simplicity and clarity only we neglect the initial conditions on g--correlations are "driven in" from $t_{p}=-\infty$ by the test particle motion alone. (This neglect will be rectified in the next section.) Thus, for $t>t_{0}$, we insert (28) into (27) to find

$$
\begin{gathered}
I_{+}\left(Y, t_{i} Y_{0}, t_{0}\right)=\sum_{S^{\prime}} \int d X^{\prime} \exp \left[-i L\left(t-t_{0}\right)\right] \delta\left(Y-X^{\prime}\right) \\
x \exp \left[-i\left(L^{\prime}+L_{0}\right) t_{0}\right]\left(n^{\prime}\right)^{-1} \delta\left(X^{\prime}-Y_{0}\right) f\left(Y_{O}\right) \\
=\sum_{S^{\prime}} \int d X^{\prime} \exp (-i L t)\left(n^{\prime}\right)^{-1} \delta\left(Y-X^{\prime}\right) \exp \left(-i L_{O} t_{0}\right) \delta\left(X^{\prime}-Y_{0}\right) f\left(Y_{0}\right) .
\end{gathered}
$$

Insel ling into (29) the identity

$$
\exp \left(-i S^{\prime} t\right) \exp \left(i S^{\prime} t\right)=1
$$


and noting that since $S$ is a differential operator

$\iint X^{\prime}\left[\exp \left(-i S^{\prime} t\right) A\left(x^{\prime}\right)\right] B\left(x^{\prime}\right)=\int d X^{\prime} A\left(x^{\prime}\right)\left[\exp \left(i S^{\prime} t\right) B\left(x^{\prime}\right)\right]$,

(29) becomes

$$
\begin{aligned}
& \Gamma_{+}\left(Y, t_{;} Y_{O^{\prime}} t_{0}\right) \doteq \sum n^{\prime} \int d X^{\prime}\left\{\exp \left[-i\left(L+S^{\prime}\right) t\right]\left(n^{\prime}\right)^{-1} \delta\left(Y-X^{\prime}\right)\right\} \\
& x\left[\exp \left(-i L_{0} t^{\prime}-i S^{\prime} t\right)\left(n^{\prime}\right)^{-1} \delta\left(X^{\prime}-Y_{0}\right) f\left(Y_{0}\right)\right] \text {. }
\end{aligned}
$$

Inserting the identity in the form

$$
\sum_{s^{\prime}} \int \mathrm{dx} \mathrm{x}^{\prime \prime} \delta\left(\mathrm{x}^{\prime \prime}-\mathrm{x}^{\prime}\right)=1
$$

(30) becomes

$$
\begin{aligned}
\Gamma_{+}\left(Y, t ; Y_{0} t_{0}\right) & \\
= & \sum n^{\prime} n^{\prime \prime} \int d X^{\prime} d X^{\prime \prime}\left\{\exp \left[-i\left(L+S^{\prime}\right) t\right]\left(n^{\prime}\right)^{-1} \delta\left(Y-X^{\prime}\right)\right\} \\
& x \exp \left(-i L_{0} t_{0}-i S^{\prime} t\right)\left(n^{\prime \prime}\right)^{-1} \delta\left(X^{\prime \prime}-X^{\prime}\right)\left(n^{\prime}\right)^{-1} \delta\left(X^{\prime}-Y_{0}\right)^{\prime} f\left(Y_{0}\right)
\end{aligned}
$$

We now write $\delta\left(\mathrm{X}^{\prime \prime}-\mathrm{X}^{\prime}\right) \delta\left(\mathrm{X}^{\prime}-\mathrm{Y}_{0}^{\prime}\right)=\delta\left(\mathrm{X}^{\prime \prime}-\mathrm{Y}_{0}\right) \delta\left(\mathrm{X}^{\prime \prime}-\mathrm{X}^{\prime}\right)$ $\exp \left(-i S^{\prime} t\right)=\exp \left[-i S^{\prime}\left(t-t_{0}\right)\right] \exp \left(-i S^{\prime} t_{0}\right)$, and integrate $\exp \left(-i S^{\prime} t_{0}\right)$ by parts, using also (14), to bring (31) to the form

$$
\begin{aligned}
& \Gamma_{+}\left(Y, t_{i} Y_{0}, t_{0}\right) \\
& =\sum n^{\prime} n^{\prime \prime} \int d x^{\prime} d x^{\prime \prime}\left\{\exp \left[-i\left(L+S^{\prime}\right) t\right]\left(n^{\prime}\right)^{-1} \delta\left(Y-X^{\prime}\right)\right\}
\end{aligned}
$$

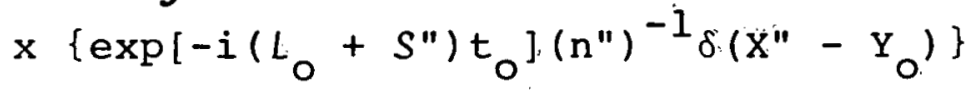

$$
\begin{aligned}
& x\left\{\exp \left[-i S^{i}\left(t-t_{0}\right)\right]\left(n^{\prime \prime}\right)^{-1} \delta\left(x^{\prime \prime}-x^{\prime}\right) f\left(x^{\prime \prime}\right)\right\} \text {. }
\end{aligned}
$$


However, using (15) and (17), (32) becomes

$$
\begin{aligned}
& \Gamma_{+}\left(Y, t_{;} Y_{0}, t_{0}\right) \\
& \quad=\sum n^{\prime} n^{\prime \prime} \int d X^{\prime} d X^{\prime \prime} Y\left(Y, t \mid X^{\prime}\right) \gamma\left(Y_{0}, t_{0} \mid X^{\prime \prime}\right) W_{+}\left(X^{\prime}, t_{i} X^{\prime \prime}, t_{0}\right),
\end{aligned}
$$

which is precisely the one-sided version of (18). Thus, the superposition principle is proved in this special case. By the replacement (20) we obtain the proof for arbitrary time dependence. The initial transients in $g$ could be included in this proof with no complication in principle. This will become clear in the next section, where we do discuss the initial conditions explicitly.

\section{A NEW INDUCTIVE PROOF}

In this section we give for completeness a simplified form of Rostoker's inductive proof: we postulate (18), then show that both sides obey the same equations, with identical boundary conditions. The proof consists of two steps. First, we assume that the "static" autocorrelation $\Gamma\left(t_{0} ; t_{0}\right) \equiv \xi\left(t_{0}\right)$ is given correctly by the r.h.s. $\hat{\Gamma}$ of (18), then verify that $\tilde{\Gamma}$ also yields correctly the dynamics $t_{0} \rightarrow t$ as described by (9). Second, we verify that $\tilde{\xi}$ also solves $(20)$ with the appropriate initial condition.

For part one, we write

$$
\begin{aligned}
\left(\partial_{t}\right. & +i L) \tilde{\Gamma}\left(Y, t_{i} Y_{O^{\prime}}, t_{0}\right) \\
= & \sum \dot{n}^{\prime} n^{\prime \prime} \int d X^{\prime} d X^{\prime \prime}\left\{\gamma\left(Y, t \mid X^{\prime}\right)\left[\partial_{t} W\left(X^{\prime} ; t_{;} X^{\prime \prime}, t_{0}\right)\right] \gamma\left(Y_{0}, t_{0} \mid X^{\prime \prime}\right)\right. \\
& \left.+\left[\left(\partial_{t}+i L\right) \gamma\left(Y, t \mid X^{\prime}\right)\right] W\left(X^{\prime}, t_{i} X^{\prime \prime}, t_{0}\right) \gamma\left(Y_{0}, t_{0} \mid X^{\prime \prime}\right)\right\} .
\end{aligned}
$$


We use (15a) to replace $\left(\partial_{t}+i L\right) \gamma$ by -iS' $\gamma$; then integrating the second term in (33) by parts, we bring the r.h.s. of (33) to. the form

$$
\sum n^{\prime} n^{\prime} \int d x^{\prime} d X^{\prime \prime} \gamma\left(y, t \mid X^{\prime}\right)\left[\left(\partial_{t}+i S^{\prime}\right) w^{\prime}\left(X^{\prime}, t ; x^{\prime \prime}, t_{0}\right)\right] \gamma\left(y_{0}, t_{0} \mid X^{\prime \prime}\right),
$$

which vanishes according to (17a). Thus,

$$
\left(\partial_{t}+i L\right) \ddot{\Gamma}=0 \text {. }
$$

which is the same dynamical equation (9a) satisfied by $\Gamma$; this implies $\Gamma=\tilde{\Gamma}$ if the initial conditions on both quantities are identical. Therefore, we must now prove as part two of the proof

$$
\begin{aligned}
& \xi\left(Y, Y_{O}, t_{O}\right)=\tilde{\xi}\left(Y, Y_{O}, t_{O}\right) \\
& \equiv \sum n^{\prime} n^{\prime \prime} \int d X^{\prime} d X^{\prime \prime} \gamma\left(Y, t_{0} \mid X^{\prime}\right) w\left(X^{\prime}, t_{0} ; X^{\prime \prime}, t_{0}\right) \gamma\left(Y_{0}, t_{0} \mid X^{\prime \prime}\right) \ldots \\
& =\sum n^{\prime \prime} \int d X^{\prime \prime} \gamma\left(Y, t_{0} \mid x^{\prime \prime}\right) f\left(x^{\prime \prime}, t_{0}\right) \gamma\left(Y_{0}, t_{0} \mid x^{\prime \prime}\right) \text {, }
\end{aligned}
$$

where in writing the last line of (34) we used (17b). Upon inserting the definitions (6) and (11) of $\xi$ and $\gamma$ and performing the integration, we see that (34) is equivalent to the assertion

$$
\begin{aligned}
g\left(Y, Y_{0}, t_{O}\right)= & P\left(\because, t_{0} \mid Y_{0}\right) f\left(Y_{O}, t_{O}\right)+f\left(Y, t_{0}\right) P\left(Y_{O}, t_{O} \mid Y\right) \\
& +\sum n " \int d X^{\prime \prime} P\left(Y, t_{O} \mid X^{\prime \prime}\right) \cdot f\left(X^{\prime \prime}, t_{O}\right) P\left(Y_{O}, t_{O} \mid X^{\prime \prime}\right) .
\end{aligned}
$$

The physical significance of this statement has been discussed by Rostoker. 1 
From (34), we have

$$
\begin{aligned}
& \left(\partial_{t}+i L+i L_{O}\right) \tilde{\xi}\left(Y, Y_{O}, t_{O}\right)
\end{aligned}
$$

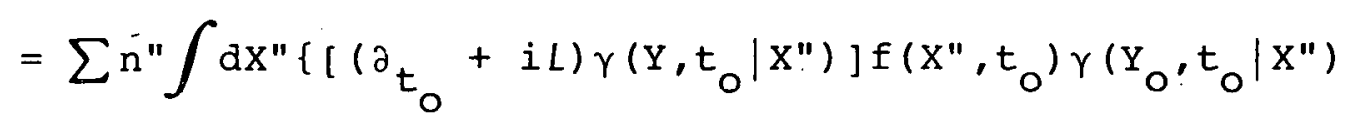

$$
\begin{aligned}
& +\gamma\left(Y, t_{0} \mid X^{\prime \prime}\right)\left[\partial_{t_{0}} f\left(X^{\prime \prime}, t_{0}\right)\right] \gamma\left(Y_{0}, t_{0} \mid X^{\prime \prime}\right) \\
& \left.+\gamma\left(Y, t_{O} \mid X^{\prime \prime}\right) f\left(X^{\prime \prime}, t_{O}\right)\left[\left(\partial_{t_{0}}+i L_{O}\right) \gamma\left(Y_{O}, t_{O} \mid X^{\prime \prime}\right)\right]\right\} .
\end{aligned}
$$

The term involving $\left(\partial_{t_{0}}+i L\right) \gamma$ may be replaced by $-i S " \gamma$ according to (15a). Then, integrating the first term of (36) by parts and noting that since $S^{\prime \prime}$ is a differential operator,

$$
S^{\prime \prime}(f \gamma)=f\left(S^{\prime \prime} \gamma\right)+\left(S^{\prime \prime} f\right) \gamma \text {. }
$$

the terms involving $S^{\prime \prime} \gamma$ cancel leaving us with

$$
\sum n^{\prime \prime} \int \mathrm{dx}^{\prime \prime} \gamma\left(y, t_{0} \mid \mathrm{X}^{\prime \prime}\right)\left[\left(\partial_{t_{0}}+i S^{\prime \prime}\right) f\left(x^{\prime \prime}, t_{0}\right)\right] \gamma\left(Y_{0}, t_{0} \mid X^{\prime \prime}\right) \text {. }
$$

But

$$
\left(\partial_{t_{0}}+i S^{\prime \prime}\right) f\left(x^{\prime \prime}, t_{0}\right)=0
$$

is just the nonlinear Vlasov equation, which $f$ must solve to this order. Thus (36) becomes

$$
\left(\partial_{L_{0}}+i \underline{L}+\dot{i} L_{U}\right) \tilde{\dot{\xi}}=0
$$

which is the same dynamical equation as (20a). We will then have 
$\tilde{\Gamma}=\Gamma$, completing our proof, if we verify that the initial condition at $t_{0}=t_{p}$ is satisfied. We must then verify

$$
\begin{aligned}
& \xi\left(Y, Y_{0}, t_{p}\right)=n^{-1} \delta\left(Y-Y_{0}\right) f\left(Y_{0}, t_{p}\right)+g\left(Y, Y_{0}, t_{p}\right) \\
& =\tilde{\xi}\left(Y, Y_{0}, t_{p}\right) \\
& =n^{-1} \delta\left(Y-Y_{0}\right) f\left(Y_{0}, t_{p}\right)+P\left(Y, t_{p} \mid Y_{0}\right) f\left(Y_{0}, t_{p}\right) \\
& +P\left(Y_{O}, t_{p} \mid Y\right) f\left(Y, t_{p}\right) \\
& +\sum_{:} n^{\prime \prime} \int d X^{\prime} P\left(Y, t_{p} \mid X^{\prime \prime}\right) f\left(X^{\prime \prime}, t_{p}\right) P\left(Y_{\circ}, t_{P} \mid X^{\prime \prime}\right) \text {. }
\end{aligned}
$$

In the usual application of the superposition principle, only correlations driven in by the streaming motion of the test particle are considered; initial transients in $g$ are neglected. For this case, $g\left(t_{p}\right)=P\left(t_{p}\right)=0$ and $(38)$ is satisfied. For the case when $g\left(t_{p}\right)$ represents "physical" correlations, $(38)$ is also satisfied. By this, we mean that we treat $t_{p}$ as simply some early time to which $g$ has evolved according to (20) from a still earlier physical state of the system (which is usually $t_{p}=-\infty, P(-\infty)=0$, as in the case of thermal equilibrium). Since we have already verified (35) for arbitrary times $t_{o^{\prime}}(39)$ is a possible initial state. We shall not be concerned with other, unphysical initial correlations. We thus conclude that (18) is satisfied, and our inductive proof of the superposition principle is complete. 


\section{DISCUSSION AND SUMMARY}

The result (8) is written for an arbitrary number of species and is thus slightly more general than that of Rostoker, who considered only the case of equal species densities. We repeat for emphasis Rostoker's observation that the principle holds for arbitrary space-time dependence of $f(x, t)$ (although this lowest order truncation in $\varepsilon_{p}$ need not provide a reasonable or adequate representation of the physics, especially if the plasma is far from thermal equilibrium).

Our $\Gamma$ approach emphasizes the fundamental vlasov character of the plasma very strongly, as we have dealt here with only the lowest order version of the williams-oberman two-time fluctuation theory. Thus, the fluctuation spectra computed from the Fourier analysis of (7) or (8) can be called "collisionless," since collisional damping of an individual fluctuation is absent. Already at this level, the power of the formalism and its advantages over the previous $\Omega, F$ theories is quite apparent. The general theory 7,8 is also well suited for deriving higher order generalizations of the superposition principle. We shall, however, reserve discussion of these generalizations and the possibility of a renormalization of the superposition principle to a future paper. Of course, the vlasov equation (9) is correct only for times short relative to the collision time. In addition, it is correct only for lengths $\ell \sim \lambda_{D}$. However, several recent theories of shorttime fluctuations 10,13 have shown that the vlasov equation remains valid for all length scales if the bare coulomb potential is replaced appropriately by the ornstein-zernike direct correlation 
function $c$. For the special case of thermal equilibrium plasma, the proper substitution is in Fourier space ${ }^{14}$

$$
\begin{aligned}
& \frac{4 \pi q_{r} q_{s}}{k^{2}} \rightarrow-T \frac{1}{n_{r}} c_{r s}(k), \\
& \underset{\approx}{c}(k) \equiv{ }_{\approx}^{n} \cdot \underset{\approx}{g}(k) \cdot \underbrace{[1}_{\approx}+\underset{\approx}{n} \cdot \underset{\approx}{g}(k)]^{-1}, \\
& n_{i=s}=n_{1 \cdot} \delta_{r s}
\end{aligned}
$$

where $\mathrm{k}$ is the wave number, $\mathrm{T}$ the temperature, the species labels $r, s$ are interpreted as matrix indices, and

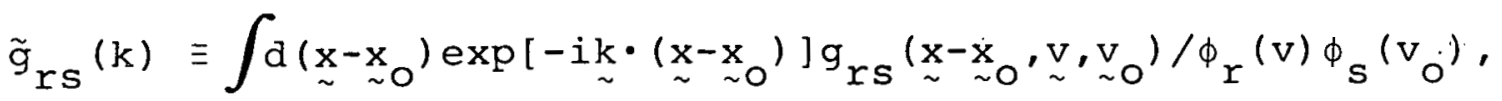

with $g$ the usual pair correlation function and $\phi_{x}$ the Maxwellian $\phi_{r}(v) \equiv\left(2 \pi v_{T r}^{2}\right)^{-d / 2} \exp \left(-v^{2} / 2 v_{T r}^{2}\right), v_{T r}^{2} \equiv T / m_{r}$. with this substitution the superposition principle remains valid for the short-time theory of neutral. fluids as well as for plasmas if the appropriate pair correlation function $g$ is given. Although the longranged nature of the coulomb force makes the short-time plasma theory of much more interest than the corresponding theory for the neutral fluid, the latter has found some applications--for example, in explaining collisionless sound in liquid lead. ${ }^{15}$ Thus, the superposition principle may be of use in contexts other than the plasma physical one in which it was first introduced. 
In summary, we have presented two new and straightforward proofs of the test particle superposition principle, beginning from the lowest order, collisionless equation for the phase space autocorrelation $\Gamma$. That a deductive proof of the superposition principle is possible was demonstrated here for the first time in the literature. Both the deductive and the inductive proofs were simpler than Rostoker's original one, as we invoked from the outset only vlasov dynamics; particle discreteness entered only as an initial condition. With the appropriate choice of pair correlation function, the superposition principle is also applicable to the short-time theory of neutral fluids.

\section{ACKNOWLEDGMEN'IS}

The author is grateful to Carl oberman for many interesting and informative discussions on the physical nature of plasma kinetic theory, and for useful comments concerning the manuscript.

This work was supported by U. S. Energy Research and Development Administration Contract E(11-1)-3073. 


\section{REFERENCES}

*Present address: Institute for Advanced Study, Princeton, New Jersey 08540 .

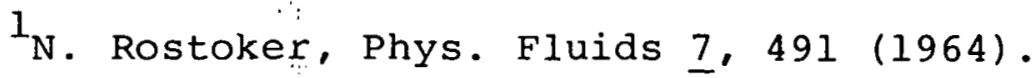

2 N. Rostoker., Nucl. Fusion 1,101 (1961).

${ }^{3}$ N. Rostoker, Phys. Fluids 7,479 (1964).

${ }^{4}$ E. A. Wiliiams, Ph.D. thesis, Princeton University. (1973).

${ }^{5}$ The only practical exception to this statement occurs in the theory of test particle motion, where $\Omega$ is the appropriate probabilistic quantity.

${ }^{6}$ As written, (2) is ambiguous since it is not clear to which variable, $X$ or $Y$, the species sum $\sum n$ belongs. All such ambiguities are to be resolved by associating the species sum with the adjoining integration variable. Strictly speaking, in fact, the species sum should be absorbed in the integration symbol according to our definition of a phase point; however, we have chosen to make the sum explicit tor clairity and emphasis.

${ }^{7} \mathrm{~J}$. A. Krommes, Ph.D. thesis, Princeton University (1975).

${ }^{8} \mathrm{~J}$. A. Krommes and C. Oberman (to be published).

${ }^{9}$ P. C. Martin, E. D. Siggia, and H. A. Rose, Phys. Rev. A8, 423 (1973).

$10_{\mathrm{J}} . \mathrm{L}$. Lebowitz, J. K. Percus, and J. Sykes, Phys. Rev. 188, 487 (1969).

${ }^{1 l_{B y}}$ "collisionless," we mean that the dynamics of $\Gamma$ are computed from the collisionless Vlasov equation. The correlation

(7) is itself $O\left(\varepsilon_{p}\right)$ and depends on particle discreteness.

${ }^{12}$ R. Kubo, J." Phys. Soc. Jap. 17, 1100 (1962). 
13 A. Z. Akasu and J. J. Duderstadt, Phys. Rev. 188, 479 (1969). D. Forster, Phys. Rev. A9, 943 (1974).

${ }^{14}$ E. J. Linnebur and J. J. Duderstadt, Plasma Phys. 15, 647 (1973).

${ }^{15}$ M. Nelkon and S. Ranganathan, Phys. Rev. 164, 222 (1967). 